\title{
Estimating a Cagan-type demand function for gold: 1561-1913
}

\author{
Alexei Deviatov and Neil Wallace
}

July 14, 2006

\begin{abstract}
Long times series on production of gold and the value of gold, taken from Jastram's book The Golden Constant, are used to estimate a Cagan-type demand function that relates the real total value of gold to its expected rate of return. The model assumes that gold production and a latent scale variable (income or consumption) are jointly exogenous and that the data are measured with error. The data reject the model: the estimates imply that the real value of gold varies a great deal relative to the expected return and depends negatively, rather than positively, on the expected return.
\end{abstract}

Key words: gold, Cagan demand function, estimation

JEL Classification \#: E41.

\section{Introduction}

A Cagan demand function for money posits that its real value depends positively on its expected return. Here, we attempt to estimate such a function for gold using data for 1561-1913. The initial date is determined by the available data, while the terminal date is chosen in accord with our view that World War I marks the beginning of a period of turmoil regarding the role of gold in private portfolios and in the world's monetary system. The data we use are from The Golden Constant by Roy Jastram [6]: a time series on the price of gold in terms of consumption (Table 3, The Index of Purchasing Power of Gold: England 1560-1976, pages 34-37) and a time series on gold production (Appendix C, The index of World Production of Gold, 
1493-1972, pages 221-225). ${ }^{1}$ Because the data describe world production of gold, we view our demand function to be one for the world as a whole.

The main challenge, of course, is modeling the expected return. Our approach is simple - perhaps, too simple. Measurement error aside, we assume that there are two exogenous random processes. One is a process for gold production and the other is a latent (unobserved by us) process for a scale variable like total income or consumption. We assume that the processes and the current state are known and that an expected return is formed based on that information and on the true demand function. ${ }^{2}$

It turns out that our model is rejected by the data in the following senses: our estimates imply that the real value of gold varies a great deal relative to the expected return and depends negatively, rather than positively, on the expected return. We hope that our effort will inspire others to consider alternative models of these time series.

\section{The data}

The gold production data are shown in Figure 1. The data prior to 1851 are averages: 20-year averages before 1811 and 10-year averages during 18111850. As described below, we use those averages and part of the model to interpolate the missing annual data on gold production.

In Figure 2 we plot the logarithm of the purchasing power of gold; that is, the logarithm of the price of gold. Notice that, in contrast to gold production, there does not seem to be a trend in the purchasing power of gold. As Jastram says, the title of his book refers to the absence of such a trend.

\section{The model}

We treat the data on gold production and the price of gold as measured with error. The following is our model for the true values. The demand function

\footnotetext{
${ }^{1}$ Jastram describes how he came to produce the volume from which we take the data: "My interest in gold began in 1936 for a pragmatic reason. As the most junior member of the Stanford University Department of Economics, I was chosen to volunteer to do some research commissioned by Mr. C.O.G. Miller, an industrialist and gentleman scholar ([6], page vii)."

${ }^{2}$ Therefore, our model is part of a large literature on "rational expectations" specifications of the Cagan model. See, for example, [2], [4], [5], [7], [8], and [9].
} 


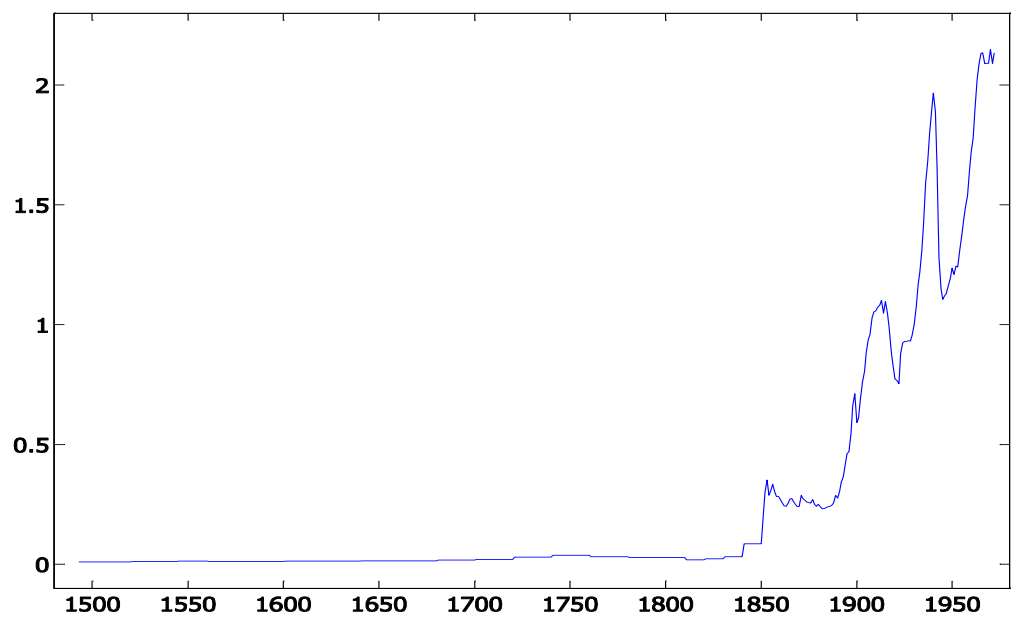

Figure 1: The Index of World Production of Gold, 1492-1972.

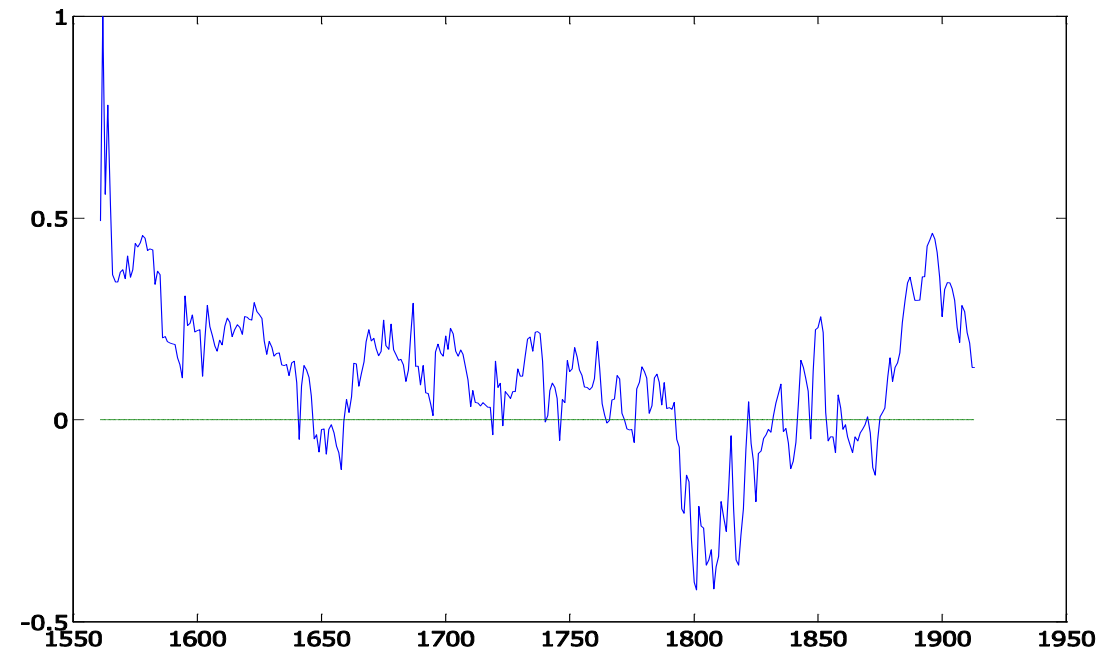

Figure 2: Logarithm of the purchasing power of gold, 1561-1913. 
is

$$
\ln G_{t}+\ln p_{t}=\ln Y_{t}+\alpha\left[E_{t} \ln p_{t+1}-\ln p_{t}\right]
$$

where $G_{t}$ is the stock of gold, $p_{t}$ is the price of gold, $Y_{t}$ is a latent (scale) variable, $\alpha$ is a constant, and $E_{t}$ denotes mathematical expectation conditional on information up through date $t$.

As noted above, there are two exogenous random processes, the date- $t$ realization of which is denoted $\left(G_{t}, Y_{t}\right)$. We assume that the process for $G_{t}$ is subject to temporary and permanent shocks, according to

$$
\ln G_{t}=\ln \left(G_{t}^{*} \gamma_{t}\right) \text { and } \ln G_{t}^{*}=\ln \left(G_{t-1}^{*} \mu_{t}\right)
$$

where $\gamma_{t}$ (the temporary shock) and $\mu_{t}$ (the permanent shock) are realizations of independent finite-state Markov processes with positive supports and where the supports for $\mu_{t}$ and for $\gamma_{t}$ are equally spaced and where that for $\gamma_{t}$ is symmetric around unity. We choose a process for $Y_{t}$ to be consistent with a stationary process for $p_{t}$. That, in turn, requires that the permanent components of $Y_{t}$ and $G_{t}$ be related. We assume that

$$
\ln Y_{t}=\ln \left(Y_{t}^{*} \eta_{t}\right) \text { and } \ln Y_{t}^{*}=\ln \left(G_{t}^{*} / \theta_{t}\right)
$$

where $\eta_{t}$ and $\theta_{t}$ are finite-state Markov processes. (The second of these is the cointegrating relationship between $Y_{t}^{*}$ and $G_{t}^{*}$ that permits there to be a stationary equilibrium.) It follows that

$$
\ln Y_{t}=\ln G_{t}^{*}+\ln \eta_{t}-\ln \theta_{t} \equiv \ln G_{t}^{*}-\ln \varsigma_{t},
$$

where $\varsigma_{t} \equiv\left(\theta_{t} / \eta_{t}\right)$ is a realization of an independent finite-state Markov processes with a positive support that is equally spaced.

Using (2)-(4), we can rewrite (1) as

$$
\ln p_{t}=-\frac{1}{1+\alpha}\left(\ln \varsigma_{t}+\ln \gamma_{t}\right)+\frac{\alpha}{1+\alpha} E_{t} \ln p_{t+1} .
$$

To give a definition of equilibrium, we need additional notation for the Markov processes; namely,

\begin{tabular}{|c|c|c|}
\hline random variable & support & transition probability \\
\hline$\gamma_{t}$ & $\left(\gamma^{1}, \gamma^{2}, \ldots, \gamma^{M_{\gamma}}\right)$ & $\pi_{i j}^{\gamma}$ \\
\hline$\mu_{t}$ & $\left(\mu^{1}, \mu^{2}, \ldots, \mu^{M_{\mu}}\right)$ & $\pi_{i j}^{\mu}$ \\
\hline$\varsigma_{t}$ & $\left(\varsigma^{1}, \varsigma^{2}, \ldots, \varsigma^{M_{\varsigma}}\right)$ & $\pi_{i j}^{\varsigma}$ \\
\hline
\end{tabular}


Then we have the following definition of a stationary equilibrium.

Definition 1 A function $p: R^{M_{\gamma}} \times R^{M_{\varsigma}} \rightarrow R_{++}$(with generic element $p_{h l}$, which denotes the price when $\left.\left(\gamma_{t}, \varsigma_{t}\right)=\left(\gamma^{h}, \varsigma^{l}\right)\right)$ is a stationary equilibrium if it satisfies

$$
\ln p_{h l}=-\frac{1}{1+\alpha}\left(\ln \varsigma^{l}+\ln \gamma^{h}\right)+\frac{\alpha}{1+\alpha} \sum_{i=1}^{M_{\gamma}} \sum_{j=1}^{M_{\varsigma}} \pi_{h i}^{\gamma} \pi_{l j}^{\varsigma} \ln p_{i j}
$$

for each $(h, l) \in\left\{1,2, \ldots, M_{\gamma}\right\} \times\left\{1,2, \ldots, M_{\varsigma}\right\}$.

This system of linear equations can be written as $x=a_{0}+a_{1} \pi x$, where $x$ is an $M_{\gamma} M_{\varsigma} \times 1$ vector, $a_{1}=\frac{\alpha}{1+\alpha}$, and $\pi$ is the Kronecker product of $\pi^{\gamma}$ and $\pi^{\varsigma}$, the transition matrices for $\gamma$ and $\varsigma$, respectively. Provided that the matrix $I-a_{1} \pi$ is nonsingular, an equilibrium exists and is unique. Obviously, that is the case if $\alpha \geq 0$. In any case, nonsingularity of the matrix $I-a_{1} \pi$ is generic.

Our basic approach to estimation follows Cosslett S. and Lee [3]. We specify magnitudes for $M_{\gamma}, M_{\mu}$, and $M_{\varsigma}$ and assume that the data are measured with multiplicative errors. Our version of the estimation procedure has two stages. ${ }^{3}$ First, we estimate the process and realization for gold production using only the data on gold production. This gives estimates of the parameters for the $\gamma_{t}$ and $\mu_{t}$ processes and for the measurement error for gold production, for the initial stock of gold, and for the realizations of the $\gamma_{t}$ and $\mu_{t}$ processes. Second, taking those as given, we estimate the rest of the model: the parameters for the $\varsigma_{t}$ process and for the measurement error in $p_{t}$, the realization for the $\varsigma_{t}$ process, the initial value of $Y_{t}$, and $\alpha$.

\section{Estimating the stock of gold}

Our model of the stock-of-gold for $t=1,2, \ldots, T$ is

$$
G_{t}=G\left(\prod_{i=1}^{t} \mu_{i}\right) \gamma_{t} \exp \left(\varepsilon_{t}^{G}\right)
$$

\footnotetext{
${ }^{3}$ See appendix 2 for a description of joint estimation of the entire model.
} 
where $G$, a parameter to be estimated, is the stock of gold at $t=0$ and $\varepsilon_{t}^{G} \sim N\left(0, \sigma_{G}^{2}\right)$, the measurement error, is i.i.d. and is independent of $\gamma_{t}, \mu_{t}$, and $\varsigma_{t}$.

We estimate some features of the Markov processes for $\gamma_{t}$ and $\mu_{t}$ and impose others. Because scaling the support of $\gamma_{t}$ by one factor and scaling the support $\mu_{t}$ by its inverse leaves the true process for gold unaffected, we normalize $\gamma_{t}$ by assuming that the support of $\gamma_{t}$ is symmetric around unity. We do not estimate the number of elements in the supports. We use $M_{\gamma}=3$ and $M_{\mu}=5$, which yield a reasonably good fit. ${ }^{4}$ And, finally, for both supports, we assume equally spaced elements; that is, $\gamma^{j+1}=\gamma^{j}+\Delta_{\gamma}$ and $\mu^{j+1}=\mu^{j}+\Delta_{\mu}$. Given the normalization imposed on the support of $\gamma_{t}$, that leaves us to estimate only $\Delta_{\gamma}$ for the support of $\gamma_{t}$. For the support of the $\mu_{t}$ process, we estimate the lower endpoint of the support, $\mu^{1}$, and $\Delta_{\mu}$. To estimate $\pi^{\gamma}$ and $\pi^{\mu}$, we use a logit representation for each row (distribution) of the transition matrices, i.e.:

$$
\pi_{i j}=\left\{\begin{array}{ll}
\frac{e^{w_{i j}}}{1+\sum e^{w_{i j}}} & \text { if } i \neq j \\
\frac{1}{1+\sum e^{w_{i j}}} & \text { if } i=j
\end{array} .\right.
$$

We impose additional bound constraints on $w_{i j}$, which ensure that the estimated transition matrices are ergodic. The latter helps us to avoid the inconvenience of having to deal with multiple stationary distributions implied by $\pi^{\gamma}$ and $\pi^{\mu}$ during the course of estimation. Thus, $\kappa=\left(G, \Delta_{\gamma}, \pi^{\gamma}, \mu^{1}, \Delta_{\mu}, \pi^{\mu}, \sigma_{G}\right)$ are the parameters to be estimated for this part of the model.

Letting $\gamma=\left\{\gamma_{t}\right\}_{t=1}^{T}$ and $\mu \equiv\left\{\mu_{t}\right\}_{t=1}^{T}$ denote possible realizations for the $\gamma_{t}$ and $\mu_{t}$ processes, the likelihood function is:

$$
L_{1}=\left[\prod_{t=1}^{T} \Psi\left[\ln G_{t}-\ln G-\sum_{i=1}^{t} \ln \mu_{i}-\ln \gamma_{t} ; \sigma_{G}\right]\right] P(\gamma) P(\mu),
$$

where $\Psi\left(x ; \sigma_{G}\right)$ is the density of the normal distribution,

$$
G_{t}=G+\sum_{i=1}^{t} Z_{i}
$$

\footnotetext{
${ }^{4}$ We arrived at these choices as follows. We began with $M_{\gamma}=3$ and $M_{\mu}=1$, which fit poorly. And we found that increasing $M_{\gamma}$ while maintaining $M_{\mu}=1 \operatorname{did}$ not substantially improve the fit. Then we tried $M_{\gamma}=3$ and $M_{\mu}=3$ and $M_{\gamma}=3$ and $M_{\mu}=5$. The latter fit substantially better than the former; as we show later, the implied measurement error $\varepsilon_{t}^{G}$ is less than $1 \%$ of the gold stock $\tilde{G}_{t}$.
} 
where $Z_{i}$ is the date $i$ datum for gold production, and where $P(\gamma)$ and $P(\mu)$ are the probabilities of the sequences $\gamma$ and $\mu$ implied by the transition matrices $\pi^{\gamma}$ and $\pi^{\mu}$.

Because the number of $\gamma$ and $\mu$ sequences is large and the state space is discrete, we use a genetic algorithm as part of our procedure for maximizing $L_{1}$. In particular, we proceed as follows.

Step 1. Generate a population of pairs of sequences which determine $\gamma$ and $\mu$, but only by their order in the respective domains. That is, the sequences corresponding to $\gamma$ are drawn from the set $\{1,2,3\}^{T}$ and those for $\mu$ are drawn from the set $\{1,2,3,4,5\}^{T}$.

Step 2. For each pair of sequences in the population of step 1, find the parameters $\kappa$ that maximize the objective $L_{1}$. (Notice that for given parameters that completes the description of the domains for $\gamma_{t}$ and $\mu_{t}-$ $\Delta_{\gamma}, \mu^{1}, \Delta_{\mu}$-a pair of sequences in the step 1 population determine $\gamma$ and $\mu$. Those realizations and the remaining parameters in $\kappa-G, \pi^{\gamma}, \pi^{\mu}, \sigma_{G}-$ determine a magnitude for $L_{1}$. Given the logit representation of the transition matrices $\pi^{\gamma}$ and $\pi^{\mu}$, the maximization problem is a standard constrained maximization problem with bound constraints. However, the objective is not concave, so there may be many local maxima.

3. Apply standard genetic operators to amend the population of step 1 sequences.

4. Repeat step 2 until the best pair $\gamma, \mu$ is found.

5. Compute confidence intervals for the estimated parameters, $\widehat{G}, \widehat{\Delta}_{\gamma}, \widehat{\mu}^{1}$, $\widehat{\Delta}_{\mu}, \widehat{\sigma}_{G}$, using a Monte Carlo procedure.

Because our concern about the post-1913 data does not apply to gold production, we use the entire data set, 1561 to 1972, for gold production. However, prior to 1851, the gold production data reported in Jastram [6] (and in the original source) are averages: twenty-year averages prior to 1811 and ten-year averages during 1811-1850. Therefore, we use an interpolation procedure, one which is consistent with the maximum likelihood estimation procedure that we carry out. Given a vector $\kappa$ of parameters and a $\gamma$ and $\mu$, the likelihood function (9) is concave with respect to the gold production data $Z_{i}$. Consequently, if some annual data are missing, then maximum likelihood interpolation of the missing data implies that the data should be interpolated in order to keep the terms,

$$
\ln G_{t}-\ln G-\sum_{\iota=0}^{t} \ln \mu_{\iota}-\ln \gamma_{t}
$$




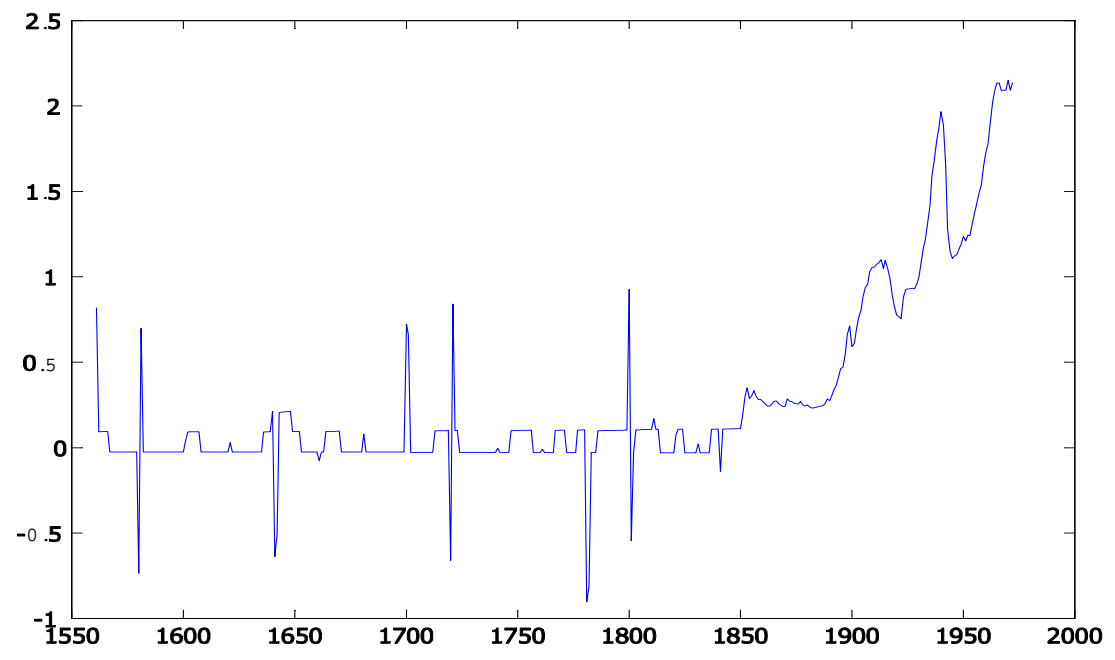

Figure 3: Gold production: interpolated up to 1850, actual after 1850.

in the likelihood function (9) constant over all sample periods for which we must interpolate. This requirement implies an interpolation procedure, one that makes the interpolated terms dependent on the $\gamma$ and $\mu$ realizations and on the parameters. Therefore, each evaluation of the likelihood function has its own interpolation of the missing data. The details appear in appendix 1.

Figure 3 contains the interpolated production series (before 1851) and the actual series (after 1851). Given the sparse parametrization (3 points in the support of $\gamma_{t}$ and 5 points in the support of $\mu_{t}$ ), a change in the state gives rise to large production changes. And because our interpolation procedure is sensitive to both permanent and temporary components (see expression 16), a change in the state creates spikes in the interpolated gold production series. 
Table 1. Estimated parameters of the gold process.

\begin{tabular}{|c|c|c|c|}
\hline \multirow{2}{*}{ Parameter } & \multirow{2}{*}{ Estimate } & \multicolumn{2}{|c|}{$5 \%$ confidence interval } \\
\cline { 3 - 4 } & & Lower end & Upper end \\
\hline$G$ & 28.5639 & 28.5085 & 29.0073 \\
\hline$\Delta_{\gamma}$ & 0.024643 & 0.024313 & 0.024835 \\
\hline$\mu^{1}$ & 0.999139 & 0.999137 & 0.999142 \\
\hline$\Delta_{\mu}$ & 0.004043 & 0.004014 & 0.004048 \\
\hline$\sigma_{G}$ & 0.002328 & 0.002151 & 0.002471 \\
\hline$L_{1}$ & 2048.33 & \multicolumn{2}{|c|}{} \\
\hline
\end{tabular}

The estimated parameters for the gold process are given in Table 1. The estimated initial stock of gold (in year 1560) equals 28.5 times the year 1930 gold production. Measured as percentages, the support of the temporary shock is approximately $\{-2.5,0,2.5\}$. The support of the permanent component is roughly $\{-0.1,0.3,0.7,1.1,1.5\}$, so that gold stock growth rates range from $-0.1 \%$ to $1.5 \%$ per year. Given our model for the gold stock (7), $\varepsilon_{t}^{G}$ measures the percentage deviation of the gold stock implied by the data from that implied by the model. The estimate $\widehat{\sigma}_{G}=0.002328$ means that the standard deviation of the discrepancy is about one quarter of one percent. In fact, for almost all of the sample, the difference between the data and the model is less than $1 \%$ and for all of the sample this difference is less than $1.3 \%$. We show the plot of $\widehat{\varepsilon}_{t}^{G}$ in Figure 4 . Notice that large spikes in interpolated production data before 1851 are consistent with small errors $\widehat{\varepsilon}_{t}^{G}$ during that period.

We use the following Monte Carlo procedure to obtain the confidence intervals in Table 1. First, we use $\widehat{\sigma}_{G}$ to generate a sequence of simulated errors $\widetilde{\varepsilon}_{t}^{G}$ from the normal distribution with zero mean and variance $\widehat{\sigma}_{G}^{2}$. After that, we use $\widehat{\gamma}, \widehat{\mu}$, and $\widehat{G}$ to compute a simulated gold stock series; namely,

$$
\widetilde{G}_{t}=\widehat{G}\left(\prod_{i=1}^{t} \widehat{\mu}_{i}\right) \widehat{\gamma}_{t} \exp \left(\widetilde{\varepsilon}_{t}^{G}\right) .
$$

Then, we treat $\widetilde{G}_{t}$ as "data" and obtain new estimates of $\gamma, \mu, G$, and $\sigma_{G}$. In doing this we keep constant the estimated sequences of states for $\gamma$ and $\mu$ by order described in step 1 above. 


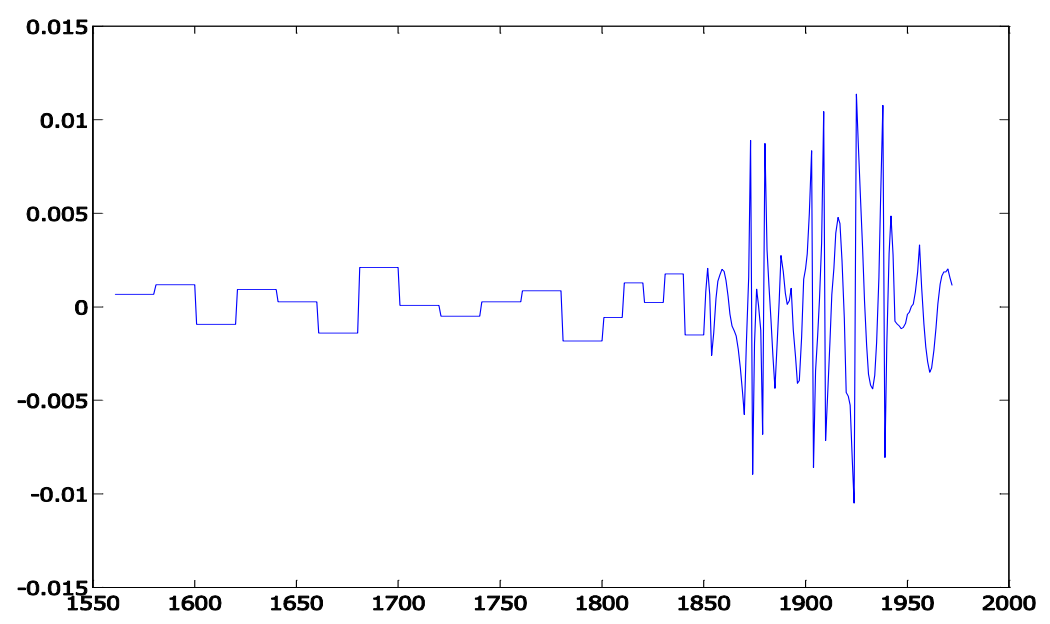

Figure 4: Estimated Gold Measurement Error $\widehat{\varepsilon}_{t}^{G}, 1561-1972$.

A more complete procedure would reestimate the sequences of states for $\gamma$ and $\mu$ by order. Such a procedure would yield larger confidence intervals. However, our estimates of the ratios $\widehat{\Delta}_{\gamma} / \widehat{\sigma}_{G} \approx 11$ and $\widehat{\Delta}_{\mu} / \widehat{\sigma}_{G} \approx 1.7$ (see Table 1) imply that only a small part of the simulated data would differ from the actual data enough to produce changes in the sequences for $\gamma$ and $\mu$ by order. Given (12), the ratios $\widehat{\Delta}_{\gamma} / \widehat{\sigma}_{G}$ and $\widehat{\Delta}_{\mu} / \widehat{\sigma}_{G}$ are effectively the t-ratios for the null hypothesis that the data distinguish between the two adjoining points in the supports of $\gamma$ and $\mu$, respectively. The ratio $\widehat{\Delta}_{\mu} / \widehat{\sigma}_{G}$, approximately equal to 1.7 , implies that under the more complete procedure only about $10 \%$ of the sequence for $\mu$ by order would differ from the estimated sequence for $\mu$. Thus, it did not seem worthwhile to undertake the more complete procedure, which would require a large amount of additional computational time. ${ }^{5}$

The $5 \%$ confidence intervals are reported in the two rightmost columns in Table 1. Notice that the estimates $\widehat{G}, \widehat{\Delta}_{\gamma}, \widehat{\mu}^{1}, \widehat{\Delta}_{\mu}, \widehat{\sigma}_{G}$ are not midpoints of those intervals. Given our procedure, the true gold stock series $G_{t}$ can be viewed as implied by the sequence of measurement errors, $\widehat{\varepsilon}_{t}^{G}$, which is drawn from the normal distribution with zero mean and variance $\widehat{\sigma}_{G}^{2}$. There

\footnotetext{
${ }^{5}$ See appendix 3 for further details.
} 
is no prior reason to expect that that drawing yields the estimates $\widehat{G}, \widehat{\Delta}_{\gamma}, \widehat{\mu}^{1}$, $\widehat{\Delta}_{\mu}$, and $\widehat{\sigma}_{G}$ which fall exactly in the middle of their $5 \%$ confidence intervals. And, as one can see, that is not the case.

The estimated transition matrices are:

$$
\widehat{\pi}^{\gamma}=\left[\begin{array}{ccc}
0.9749 & 0.0251 & 0 \\
0.1140 & 0.7170 & 0.1690 \\
0 & 0.0223 & 0.9777
\end{array}\right]
$$

and

$$
\widehat{\pi}^{\mu}=\left[\begin{array}{ccccc}
0.9264 & 0.0736 & 0 & 0 & 0 \\
0.1074 & 0.8406 & 0.0520 & 0 & 0 \\
0.0273 & 0.1131 & 0.7612 & 0.0984 & 0 \\
0 & 0 & 0.1047 & 0.7398 & 0.1555 \\
0 & 0 & 0 & 0.0814 & 0.9186
\end{array}\right] .
$$

The plots of the estimated sequences $\widehat{\gamma}$ and $\widehat{\mu}$ are given in Figures 5 and 6 . The estimates are consistent with high persistence in the gold stock process, so that the most likely state next year is the current state. However, when the transition occurs, the state does not change much; almost all changes are changes to an adjoining state. Furthermore, consistent with a considerable increase in gold production during the course of history (see Figure 1), the estimated sequence $\widehat{\mu}$ starts out low and transits to the upper end of the support.

\section{Estimating the rest of model}

Our model of the gold price data is

$$
p_{t}=p\left(\varsigma_{t}, \gamma_{t}\right) \exp \left(\varepsilon_{t}^{p}\right)
$$

where $p(\cdot, \cdot)$ is the equilibrium price function and where $\varepsilon_{t}^{p} \sim N\left(0, \sigma_{p}^{2}\right)$ is i.i.d. and is independent of $\gamma_{t}, \mu_{t}, \varsigma_{t}$, and of $\varepsilon_{t}^{G}$. As above, we interpret $\varepsilon_{t}^{p}$ as measurement error. We take $M_{\varsigma}=3$ and assume an equally-spaced support for $\varsigma_{t},\left(\varsigma^{j+1}=\varsigma^{j}+\Delta_{\varsigma}\right)$. We also treat as known the estimated stage- 1 parameters and the realizations for the $\gamma_{t}$ and $\mu_{t}$ processes. These are denoted $\hat{\kappa}, \widehat{\gamma}$ and $\widehat{\mu}$. The remaining parameters are:

$$
\rho=\left(\alpha, \varsigma^{1}, \Delta_{\varsigma}, \pi^{\varsigma}, \sigma_{p}\right) .
$$




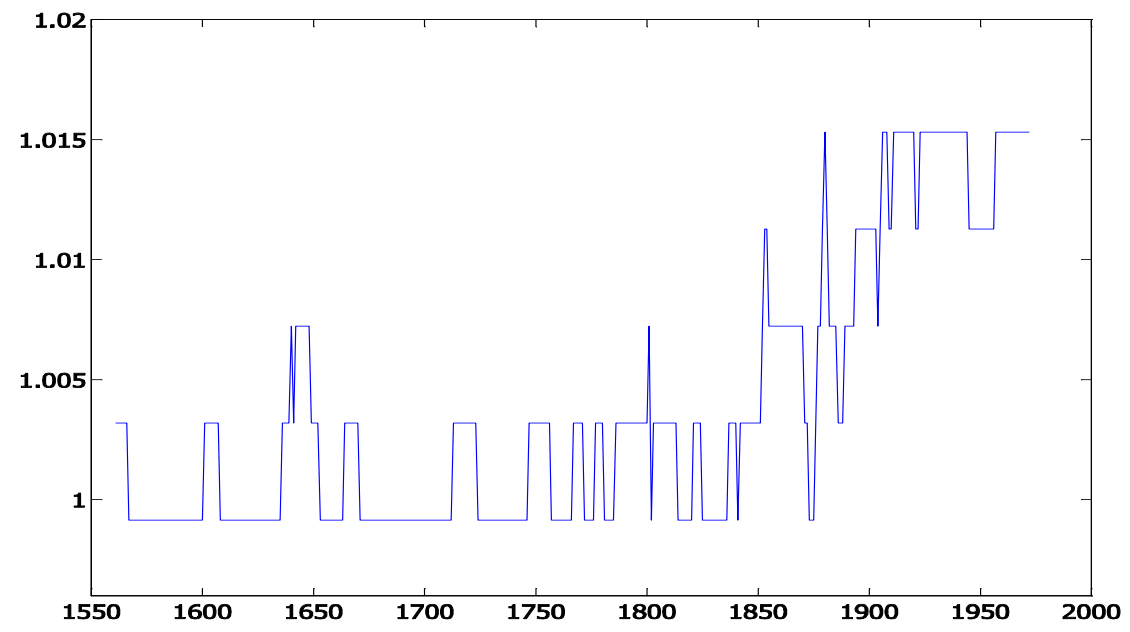

Figure 5: Estimated sequence for the permanent component of the gold stock, $\widehat{\mu}, 1561-1972$.

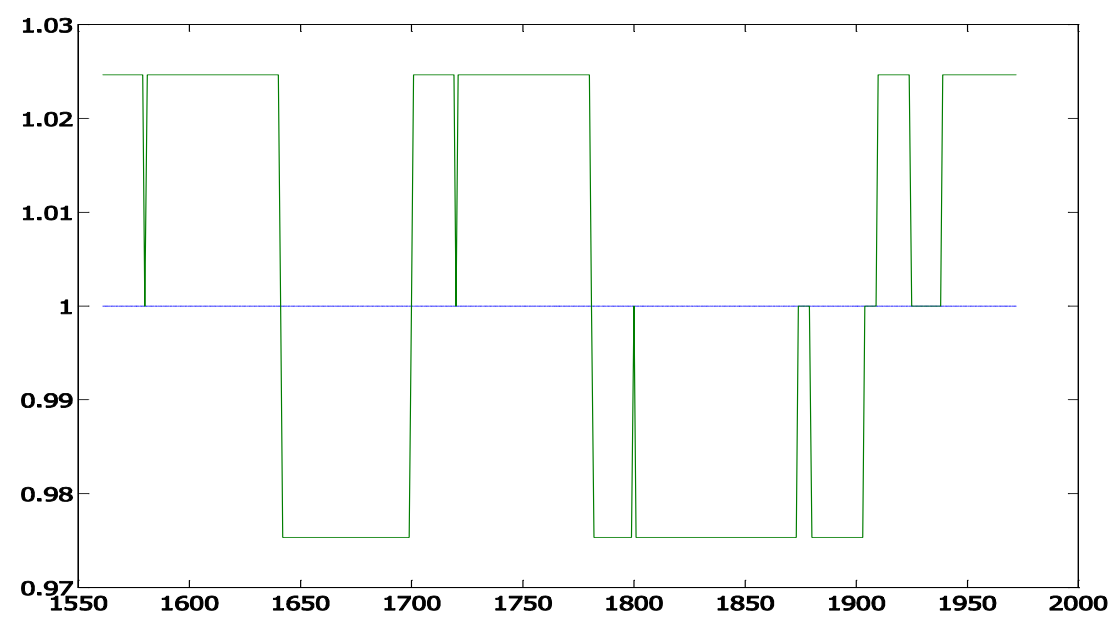

Figure 6: Estimated sequence for the temporary component of the gold stock $\widehat{\gamma}, 1561-1972$. 
Letting $\varsigma=\left\{\varsigma_{t}\right\}_{t=1}^{T}$ denote a realization for the $\varsigma_{t}$ process, the likelihood function is:

$$
L_{2}=\left[\prod_{t=1}^{T} \Psi\left[\ln p_{t}-\ln \left(p\left(\varsigma_{t}, \widehat{\gamma} ; \rho, \hat{\kappa}\right)\right) ; \sigma_{p}\right]\right] P(\varsigma),
$$

where $\Psi\left(x ; \sigma_{p}\right)$ is the density of the normal distribution, $p(\cdot, \cdot ; \rho, \hat{\kappa})$ is the solution for the equilibrium price function corresponding to the parameters $(\hat{\kappa}, \rho)$, and $P(\varsigma)$ is the probability of the sequence $\varsigma$ implied by the transition probabilities $\pi^{\varsigma}$.

Our procedure is similar to what we did when estimating the production process.

1. Generate a population of sequences which determine $\varsigma$, but only by order in the respective domain. That is, the sequences corresponding to $\varsigma$ are drawn from the set $\{1,2,3\}^{T}$.

2. For each sequence in the population of step 1, find the parameters $\rho$ that maximize the objective $L_{2}$. This involves the following steps. Pick $\rho$. This $\rho$ and a step- 1 sequence imply a corresponding sequence $\varsigma$. Solve for the equilibrium prices. Together, these imply a magnitude for $L_{2}$. Search over values of $\rho$. Given a logit representation for the transition probabilities in $\pi^{\varsigma}$, search over $\rho$ is a standard constrained maximization problem with bound constraints.

3. Apply standard genetic operators to amend the population of step 1 sequences.

4. Repeat step 2 until the best $\varsigma$ is found.

5. Compute confidence intervals using a Monte Carlo procedure.

Table 2. Estimated parameters for the rest of the model.

\begin{tabular}{|c|c|c|c|}
\hline \multirow{2}{*}{ Parameter } & \multirow{2}{*}{ Estimate } & \multicolumn{2}{|c|}{$5 \%$ confidence interval } \\
\cline { 3 - 4 } & & Lower end & Upper end \\
\hline$\alpha$ & -90.791 & -90.907 & -88.881 \\
\hline$\varsigma^{1}$ & $8.9507 \cdot 10^{-3}$ & $8.8387 \cdot 10^{-3}$ & $9.0606 \cdot 10^{-3}$ \\
\hline$\Delta_{\varsigma}$ & $1.7431 \cdot 10^{-7}$ & $6.3120 \cdot 10^{-8}$ & $2.9184 \cdot 10^{-7}$ \\
\hline$\sigma_{p}$ & 0.11244 & 0.10323 & 0.12014 \\
\hline$L$ & 557.68 & \multicolumn{2}{|c}{} \\
\hline
\end{tabular}


As noted above, we here use the sample period from 1561 to 1913 . The estimates $\widehat{\alpha}, \widehat{\varsigma}^{1}, \widehat{\Delta}_{\varsigma}, \widehat{\sigma}_{p}$ are given in Table 2 and the estimated transition matrix is

$$
\widehat{\pi}^{\varsigma}=\left[\begin{array}{lll}
0.9922 & 0.0040 & 0.0038 \\
0.0032 & 0.9930 & 0.0038 \\
0.0038 & 0.0037 & 0.9925
\end{array}\right] .
$$

The confidence intervals in Table 2 are computed using the same procedure we used above. In particular, we kept the order sequence corresponding to $\widehat{\varsigma}$ fixed during the Monte Carlo simulation of the confidence intervals. Because the change in the equilibrium price function associated with a change in the discrete order sequence for $\varsigma$, is about $21 \%$, i.e. roughly $2 \widehat{\sigma}_{p}$, only a small part of the simulated price data would differ from the actual series enough to produce changes in the discrete sequence for $\varsigma$ if we let the sequence by order change in the course of simulations. Thus, as above, it did not seem worthwhile to undertake the more complete procedure. ${ }^{6}$

The estimated $\alpha$ is large, significant, and has the wrong sign. Because the number of states $M_{\varsigma}$ is relatively small, we have not obtained a close fit of the price data; the standard deviation $\widehat{\sigma}_{p}$ of the error is quite large (about $11 \%)$. We show the actual and the fitted price in Figure 7.

The estimated sequence $\widehat{\varsigma}$ is persistent, so that the implied price $p_{t}$ is persistent as well. Persistence means that the most likely price tomorrow is the current price. In other words, high persistence implies that the expected rate of return on gold is often zero.

We present the plot of the net expected rate of return on gold in Figure 8 along with the actual return from the data, the first difference of the (logarithm of the) series in Figure 2. At no time does the net expected rate of return implied by the model exceed $0.3 \%$ in absolute value. Given the erratic pattern of actual returns, it is, perhaps, not surprising that our model gives rise to little variation in the expected return.

In Figures 9 and 10, we present two scatter plots. Figure 9 is the data on the value of the stock of gold (except that we use for the gold stock the interpolated stock [see figure 3] prior to 1850) and the return on gold. Figure 10 is the analogue using the predictions of the model. It is evident

\footnotetext{
${ }^{6}$ Again, see appendix 3 for further details.
} 


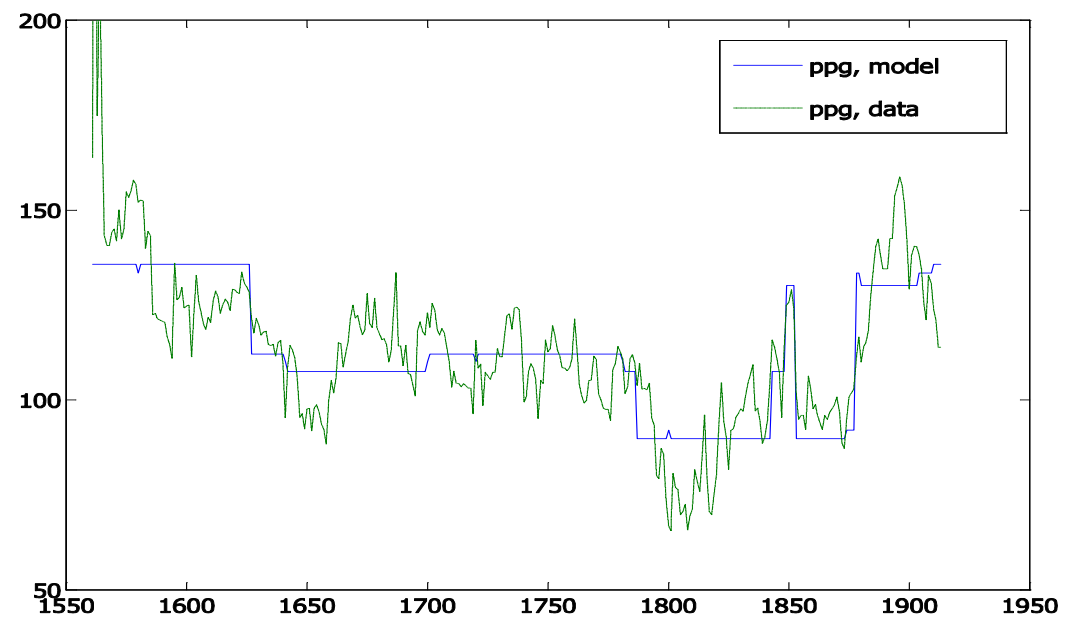

Figure 7: Purchasing power of gold: actual and fitted: 1561-1913.

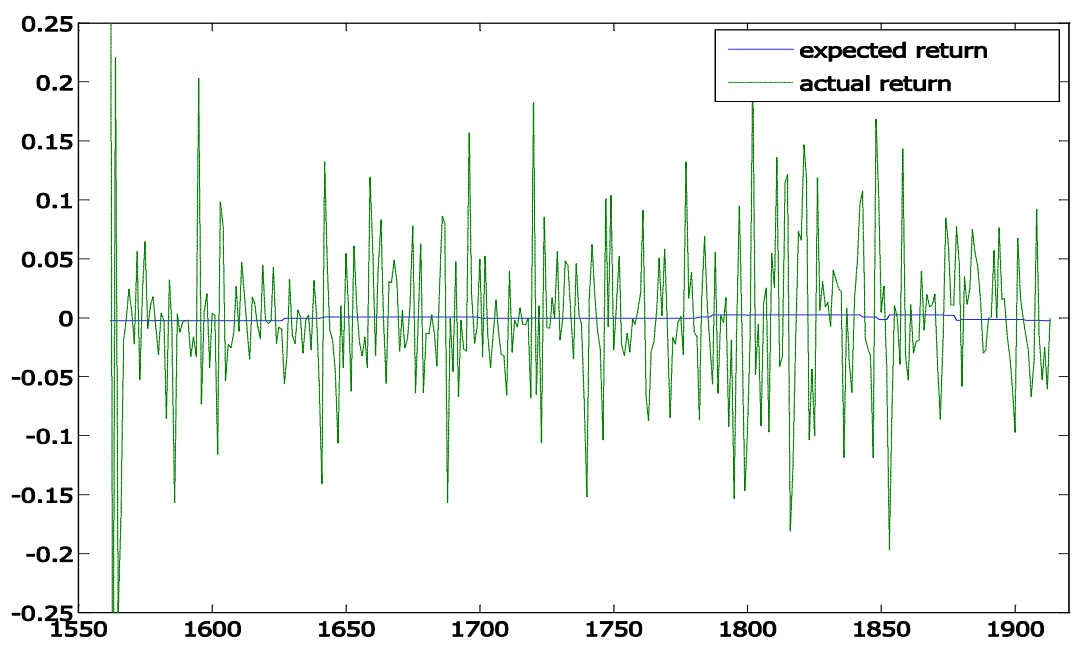

Figure 8: Actual and expected rates of return on gold, 1562-1913. 


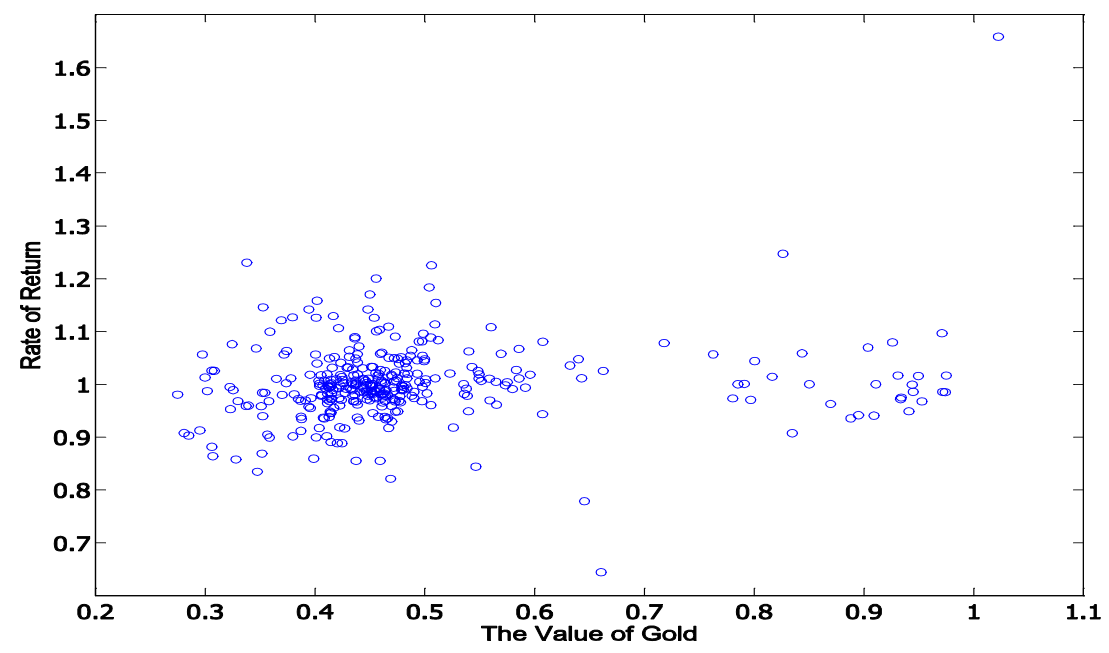

Figure 9: The value of the gold stock and the realized rate of return on gold. Actual data, 1562-1913.

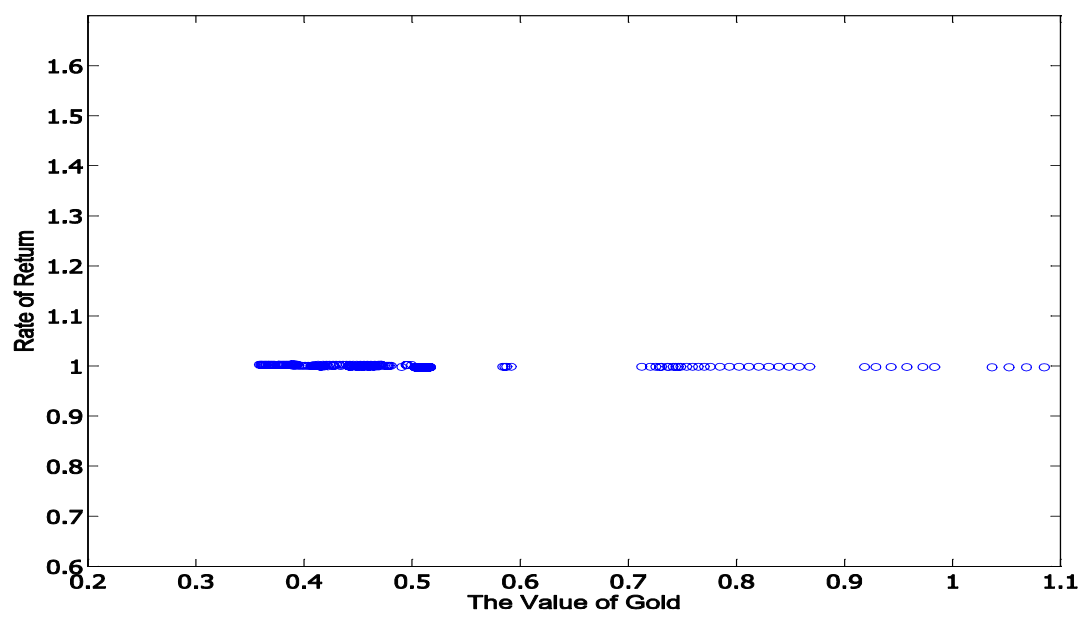

Figure 10: The value of the gold stock and the expected rate of return on gold. Fitted data, 1561-1913. 
from Figure 10 that the model implies huge sensitivity of the value of the gold stock to the rate of return, sensitivity which happens to be of the wrong sign. However, even if it were the right sign, its magnitude would be implausible.

\section{Concluding remarks}

Cagan, of course, fitted his demand function to periods of hyperinflation, when there were large "predictable" variations in the return on moneyvariations, which he argued, would swamp any changes in a scale variable like total income or consumption and any changes in the yields on alternative assets like the real return on capital. We cannot make that argument. Instead, we use his approach mainly because we do not have data on a scale variable or on the yields of alternative assets.

\section{Appendix 1. Interpolation of gold produc- tion}

Let $\left\{t_{n}+1, t_{n}+2, \ldots, t_{n+1}\right\}$ be a list of years in the sample period for which only aggregate gold production datum $\widehat{Z}_{n+1}$ is available. Constancy of all terms (11) implies that

$$
\ln G_{t+1}-\ln G_{t}=\ln \mu_{t+1}+\ln \gamma_{t+1}-\ln \gamma_{t},
$$

for $t=t_{n}+1, \ldots, t_{n+1}$. Taking (10) into account, the interpolated sequence of gold production data satisfies

$$
\widetilde{Z}_{t+1}=\left(\Upsilon_{t+1}-1\right)\left(G_{t_{n}}+\sum_{\iota=0}^{t} \widetilde{Z}_{\iota}\right),
$$

where

$$
\Upsilon_{t+1} \equiv \mu_{t+1} \frac{\gamma_{t+1}}{\gamma_{t}}
$$

Because the sequence of interpolated data $\widetilde{Z}_{t}$ must sum to $\widehat{Z}_{n+1}$, we have

$$
G_{t_{n}}+\widehat{Z}_{n+1}=\left(G_{t_{n}}+\widetilde{Z}_{t_{n}+1}\right) \prod_{i=2}^{t_{n+1}-t_{n}} \Upsilon_{t_{n}+i}
$$


It follows that

$$
\widetilde{Z}_{t_{n}+1}=\frac{G_{t_{n}}+\widehat{Z}_{n+1}}{\prod_{i=2}^{t_{n+1}-t_{n}} \mu_{t_{n}+i} \frac{\gamma_{t_{n+1}}}{\gamma_{t_{n}+1}}}-G_{t_{n}}
$$

and

$$
\widetilde{Z}_{t_{n}+s}=\left(G_{t_{n}}+\widehat{Z}_{n+1}\right) \frac{\mu_{t_{n}+s} \frac{\gamma_{t_{n}+s}}{\gamma_{t_{n}+s-1}}-1}{\prod_{i=s+1}^{t_{n+1}-t_{n}} \mu_{t_{n}+i} \frac{\gamma_{t_{n+1}}}{\gamma_{t_{n}+s-1}}}
$$

for all $s=2,3, \ldots, t_{n+1}$.

Notice that because the states $\gamma_{t}$ and $\mu_{t}$ are discrete, a switch in the estimated state can cause a large change in the interpolated data. In particular, if there is a downward switch in the state $\gamma_{t}$, then the corresponding interpolated datum $\widetilde{Z}_{t}$ can be negative. This can formally be interpreted as a loss of gold occurring at the respective dates.

\section{Appendix 2. A one-stage estimation pro- cedure}

We could estimate the entire model jointly. Let

$$
L_{3}=\prod_{t=0}^{T} \Psi\left[\ln G_{t}-\ln G-\sum_{\iota=0}^{t} \mu_{\iota}-\ln \gamma_{t} ; \sigma_{G}\right] \Psi\left[\ln p_{t}-\ln \left(p\left(\varsigma_{t}, \gamma_{t}\right)\right) ; \sigma_{p}\right]
$$

Then, given a triplet of sequences $\mu, \gamma$, and $\varsigma$, we could search for the $(\kappa, \rho)$ that maximizes

$$
L_{3} P(\mu) P(\gamma) P(\varsigma)
$$

Then, we could apply the genetic algorithm to find the optimal triplet of sequences $\mu, \gamma$, and $\varsigma$. Because some parameters - e.g. $\gamma_{t}$-appear in both parts of $L_{3}$, this joint procedure would not give the same estimates and would seem to be more efficient. However, the joint estimation is harder to carry out because of the larger set of parameters that must be searched over. 


\section{Appendix 3. Confidence intervals}

Taking the logarithm of (12) yields:

$$
\ln \widetilde{G}_{t}=\ln \widehat{G}+\sum_{i=1}^{t} \ln \widehat{\mu}_{i}+\ln \widehat{\gamma}_{t}+\widetilde{\varepsilon}_{t}^{G}
$$

which is our "data" generating process. Then, given $\widehat{\kappa}$, and the estimated pair of sequences for $\gamma$ and $\mu$ by order, the (logarithm of the) fitted gold stock series is:

$$
\omega_{t} \equiv \ln \widehat{G}+\sum_{i=1}^{t} \ln \widehat{\mu}_{i}+\ln \widehat{\gamma}_{t}
$$

Then, conditional on $\widehat{\kappa}$ the test between the two alternatives $H_{0}:(\gamma, \mu)$ and $H_{1}:\left(\gamma^{\prime}, \mu^{\prime}\right)$ is the test between the sequence $\omega \equiv\left\{\omega_{t}\right\}_{t=1}^{T}$ of fitted gold stock implied by $(\gamma, \mu)$, and the sequence $\omega^{\prime} \equiv\left\{\omega_{t}^{\prime}\right\}_{t=1}^{T}$ implied by $\left(\gamma^{\prime}, \mu^{\prime}\right)$. Given normality of $\widetilde{\varepsilon}_{t}^{G}$ the test of the alternative against the null is the test of $H_{1}: \omega^{\prime}$ against $H_{0}: \omega$, which is an F-test, while for every given date $t$ the test that $\omega_{t}^{\prime}$ differs from $\omega_{t}$ is a t-test. Given (18) the t-statistics for the test that one can distinguish between the two adjoining states $\widehat{\mu}^{j}$ and $\widehat{\mu}^{j+1}$ is

$$
\widehat{t}_{\mu}=\frac{\ln \widehat{\mu}^{j+1}-\ln \widehat{\mu}^{j}}{\widehat{\sigma}_{G}}=\frac{\ln \left(\widehat{\mu}^{1}+j \widehat{\Delta}_{\mu}\right)-\ln \left(\widehat{\mu}^{1}+(j-1) \widehat{\Delta}_{\mu}\right)}{\widehat{\sigma}_{G}} \approx \frac{\widehat{\Delta}_{\mu}}{\widehat{\sigma}_{G}} .
$$

The value of $\widehat{\Delta}_{\mu} / \widehat{\sigma}_{G} \approx 1.7$ implies that there is a roughly $10 \%$ chance that simulations alter a single entry in the sequence for $\mu$ by order. Thus, one should expect that about $10 \%$ of that sequence will differ if one allows for the more complete procedure.

As regards confidence intervals in the second stage, the "data" generating process is:

$$
\ln \widetilde{p}_{t}=\ln \widehat{p}_{t}+\widetilde{\varepsilon}_{t}^{p}
$$

where

$$
\widehat{p}_{t} \equiv p\left(\widehat{\varsigma}_{t}, \widehat{\gamma}_{t}\right)
$$

is the estimated equilibrium price function. Notice that by definition (see 6) the equilibrium price function $p\left(\varsigma_{t}, \gamma_{t}\right)$ is not necessarily a monotone function of $\varsigma$. Consequently, the t-statistics for the test that one can distinguish 
between the two adjoining values (pertaining to distinct points in the support of $\varsigma$ ) of the equilibrium price function is:

$$
\widehat{t}_{p}=\frac{\ln \widehat{p}^{j+1}-\ln \widehat{p}^{j}}{\widehat{\sigma}_{p}} \approx \frac{\frac{\hat{p}^{j+1}}{\widehat{p}^{j}}-1}{\widehat{\sigma}_{p}} .
$$

Given the estimates in Table 2, the value of $\widehat{t}_{p}$ is approximately equal to 2 , which implies that there is a roughly $5 \%$ chance that simulations alter a single entry in the estimated equilibrium price function. Thus, one should expect that about $5 \%$ of the sequence for $\varsigma$ will differ if one allows for the more complete procedure in the second stage.

\section{References}

[1] Cagan, P., The monetary dynamics of hyperinflation, in Friedman, M. (ed.), Studies in the Quantity Theory of Money, (University of Chicago Press), 1956.

[2] Christiano, L., Cagan's model of hyperinflation under rational expectations. International Economic Review 28 (1987) 33-49.

[3] Cosslett, S. and Lee, L., Serial correlation in latent discrete variable models, Journal of Econometrics 27 (1985) 79-97.

[4] Engsted, T., Cointegration and Cagan's model of hyperinflation under rational expectations. Journal of Money, Credit and Banking 25 (1993) $350-60$.

[5] Goodfriend, M., An alternative method of estimating the Cagan money demand function in hyperinflation under rational expectations. Journal of Monetary Economics 9 (1982) 43-57.

[6] Jastram, Roy W., The Golden Constant, (John Wiley and Sons), 1977.

[7] Salemi, M. and Sargent, T.J., The demand for money during hyperinflations under rational expectations, II. International Economic Review 20 (1979) 741-58.

[8] Sargent, T.J., The demand for money during hyperinflations under rational expectations, I. International Economic Review 18 (1977) 59-82. 
[9] Taylor, M.P., The hyperinflation model of money demand revisited. Journal of Money, Credit and Banking 23 (1991) 327-51. 